\title{
Myocardial scarring on cardiovascular magnetic resonance in asymptomatic or minimally symptomatic patients with "pure" apical hypertrophic cardiomyopathy
}

Kyung-Hee Kim', Hyung-Kwan Kim", In-Chang Hwang ${ }^{1}$, Seung-Pyo Lee ${ }^{1}$, Eun-Ah Park², Whal Lee ${ }^{2}$, Yong-Jin Kim¹, Jae-Hyung Park ${ }^{2}$ and Dae-Won Sohn ${ }^{1}$

\section{Abstract}

Background: Late gadolinium enhancement (LGE) cardiovascular magnetic resonance (CMR) enables state-of-the -art in vivo evaluations of myocardial fibrosis. Although LGE patterns have been well described in asymmetrical septal hypertrophy, conflicting results have been reported regarding the characteristics of LGE in apical hypertrophic cardiomyopathy (ApHCM). This study was undertaken to determine 1) the frequency and distribution of LGE and 2) its prognostic implication in ApHCM.

Methods: Forty patients with asymptomatic or minimally symptomatic pure ApHCM (age, 60.2 \pm 10.4 years, 31 men) were prospectively enrolled. LGE images were acquired using the inversion recovery segmented spoiled-gradient echo and phase-sensitive inversion recovery sequence, and analyzed using a 17-segment model. Summing the planimetered LGE areas in all short axis slices yielded the total volume of late enhancement, which was subsequently presented as a proportion of total LV myocardium (\% LGE).

Results: Mean maximal apical wall thickness was $17.9 \pm 2.3 \mathrm{~mm}$, and mean left ventricular (LV) ejection fraction was $67.7 \pm 8.0 \%$. All but one patient presented with electrocardiographic negative T wave inversion in anterolateral leads, with a mean maximum negative T wave of $7.2 \pm 4.7 \mathrm{~mm}$. Nine patients $(22.5 \%)$ had giant negative T waves, defined as the amplitude of $\geq 10 \mathrm{~mm}$, in electrocardiogram. LGE was detected in 130 segments of 30 patients (75.0\%), occupying $4.9 \pm 5.5 \%$ of LV myocardium. LGE was mainly detected at the junction between left and right ventricles in 12 (30\%) and at the apex in 28 (70\%), although LGE-positive areas were widely distributed, and not limited to the apex. Focal LGE at the non-hypertrophic LV segments was found in some ApHCM patients, even without LGE of hypertrophied apical segments. Over the 2-year follow-up, there was no one achieving the study end-point, defined as all-cause death, sudden cardiac death and hospitalization for heart failure.

Conclusions: LGE was frequently observed not only in the thickened apex of the heart but also in other LV segments, irrespective of the presence or absence of hypertrophy. The simple presence of LGE on CMR was not representative of adverse prognosis in this population.

Keywords: Apical hypertrophic cardiomyopathy, Cardiovascular magnetic resonance, Late gadolinium enhancement

\footnotetext{
* Correspondence: cardiman73@gmail.com

'Division of Cardiology, Department of Internal Medicine, Cardiovascular Center, Seoul National University College of Medicine, Seoul National University Hospital, 28 Yongon-dong, Chongno-gu, Seoul 110-744, South Korea

Full list of author information is available at the end of the article
} 


\section{Background}

Apical hypertrophic cardiomyopathy (ApHCM) is a unique phenotype of hypertrophic cardiomyopathy (HCM) with a pathologic hypertrophy of the left ventricular (LV) apex and an "ace-of-spade" configuration of the LV cavity at end-diastole by echocardiography or cardiovascular magnetic resonance (CMR). Despite a relatively favorable prognosis for ApHCM, severe clinical manifestations including arrhythmias and life-threatening apical aneurysm have been occasionally reported [1]. Although transthoracic echocardiography has been traditionally used for diagnosing HCM, the diagnosis of ApHCM by echocardiography is sometimes challenging. In this special setting, CMR can provide additional, more accurate information on LV morphology [2]. In addition, the recent introduction of late gadolinium enhancement (LGE) CMR allows the accurate detection of myocardial fibrosis in an in vivo setting [3]. According to several earlier literatures, LGE is frequently observed in asymmetrical septal HCM patients, and is also closely associated with severe hypertrophy and a high risk of sudden cardiac death [3-6]. However, LGE features of CMR in ApHCM have rarely been reported, and moreover results disagree regarding LGE patterns in ApHCM $[4,7,8]$. Therefore, this study was set out to determine the frequency and distribution of LGE on contrast-enhanced CMR in ApHCM. In addition, based on close follow-up conducted over 2 years, we sought to determine whether LGE on CMR has prognostic impact in this population.

\section{Method}

\section{Study population}

In this prospective study, we enrolled 40 asymptomatic or minimally symptomatic ApHCM patients (31 men; average age 60.2 \pm 10.4 years) scheduled for CMR in our institution. Diagnosis of ApHCM was established based on the demonstration of asymmetrical LV hypertrophy confined to the LV apex, an apical wall thickness $\geq 15$ $\mathrm{mm}$, and a maximum apical thickness to maximum basal thickness ratio of $\geq 1.3$ by CMR. Great care was taken to enroll ApHCM patients with involvement of the apex only $[9,10]$. All patients were evaluated with invasive or computed tomographic coronary angiography for the presence of coronary artery disease, and patients with significant stenosis, defined as luminal stenosis of $>50 \%$, were systematically excluded. Patients who were not in sinus rhythm were excluded, as well. The study protocol was approved by the institutional review board of our hospital and informed consent was obtained from all participants before enrollment.

\section{Electrocardiography}

A "giant" negative $\mathrm{T}$ wave in electrocardiogram (ECG) was defined as a voltage of negative $T$ wave of $\geq 1 \mathrm{mV}$ $(\geq 10 \mathrm{~mm})$ in any anterior lead. Maximum $\mathrm{T}$ wave inversion from leads V3, V4, V5, and V6 and summed T wave amplitudes in these leads were compared.

\section{Echocardiography}

All echocardiographic examinations were performed on the same day as CMR using a commercially available machine (Vivid 7, GE, Horten, Norway) with subjects in the left lateral decubitus position. A routine standard echocardiographic examination, which included measurements of LV systolic and diastolic functions, was performed. In each case, maximal apical wall thickness at end-diastole was determined using the standard apical four-, two-, and three-chamber views.

\section{CMR protocol}

CMR was performed using a $1.5 \mathrm{~T}$ scanner (Sonata Magnetom, Siemens, Erlangen, Germany). Steady-state free precession cine images were used to quantify LV function (slice thickness of $6 \mathrm{~mm}$ ), and LV short-axis images were acquired from apex to base to cover entire LV volumes. Repeated breath-holds were required in order to create adequate images. The temporal resolution was 25-30 frames per RR interval. LV mass was measured with commercially available software (QMASS MR; Medis, Leiden, Netherlands) by a single experienced observer unaware of echocardiographic data. LGE images were acquired 10 minutes after the intravenous administration of gadopentetatedimeglumine $(0.15 \mathrm{mmol} / \mathrm{kg}$ intravenous injection, Magnevist; Schering, Berlin, Germany), and followed by a flush of $20 \mathrm{~mL}$ of saline at the same rate. LGE images from long-axial, short-axial, and 4-chamber views were acquired using the inversion recovery segmented spoiled-gradient echo and phasesensitive inversion recovery methods. LGE images were evaluated using a 17-segment model, as suggested by the American Heart Association criteria [11]. LV was evaluated in the short-axis images of basal, mid, and apical segments. Basal and mid cavities were divided into 6 equal segments: anterior, anteroseptal, inferoseptal, inferior, inferolateral, and anterolateral. Apical segments were divided into 4 segments: anterior, septal, inferior, and lateral. Apical cap constituted the $17^{\text {th }}$ segment of LV myocardium.

\section{Data analysis}

Imaging data were analyzed with a commercially available postprocessing workstation (cmr42, Circle Cardiovascular Imaging Inc., Calgary, Canada). Endocardial and epicardial contours were traced manually on short-axis cine MR images of the LV at end diastole and end systole to assess end-diastolic and end-systolic volumes, LV ejection fraction, and LV mass. In order to assess the presence or absence of LGE and its extent, all short-axis 
slices from base to apex were inspected visually to identify areas of normal (completely nulled) myocardium. LGE was considered present when the signal intensity of the index myocardial segment was greater than 6SD, as compared with the remote normal myocardial signal $[12,13]$. Summing the planimetered areas of LGE in all short-axis slices yielded total volume of delayed enhancement, which was subsequently expressed as a proportion of total LV myocardium (\% LGE). Contrast-enhanced images were analyzed by two experienced observers unaware of other CMR, echocardiographic, and clinical data (K.H.K., I.C.H.). Any discrepancy in analysis between the 2 readers was resolved by a senior observer (W.L.). Additionally, LGE in each of the 17 segments was graded using a 2-point scale (segmental fibrosis score; $0=$ absence of LGE, $1=$ presence of LGE), if CMR LGE was present using the 6SD method. Figures 1 and 2 are representative CMR examples of patients without and with diffuse LGE, respectively.

\section{Follow-up}

In order to determine the impact of CMR-detected LGE on prognosis, ApHCM patients were clinically followed every 3 or 6 months for at least 2 years at a specialized HCM clinic by one cardiologist (K.H.K.). No patient was lost to follow-up. The study endpoint was a composite of all-cause mortality, sudden cardiac death, and hospitalization for heart failure.

\section{Statistical analysis}

Data are presented as mean \pm SD for continuous variables and as numbers (\%) for categorical variables, as appropriate. After confirming the normality of the distributions of continuous variables using the ShapiroWilk test, the unpaired Student t-test was used to analyze normally distributed variables, and the MannWhitney $U$ test to analyze non-normally distributed variables. Spearman correlation analysis was used to explore the possibilities of correlations between LV maximal wall thickness, ECG-determined $\mathrm{T}$ wave amplitudes. All statistical analyses were performed using statistical software package for windows (SPSS 17.0, SPSS Inc.) and a $\mathrm{p}$ value of $<0.05$ was considered statistically significant.

\section{Results}

\section{Clinical characteristics}

Baseline clinical and demographic characteristics of the 40 ApHCM patients are illustrated in Table 1. Ages ranged from 28 to 80 years (median, 5 9years). There were 31 men and 9 women. Of the 40 patients, 25 (63.5\%) were completely asymptomatic and 15 had only mild symptoms (New York Heart Association class I or II) at the time of study enrollment. The primary reason for initial echocardiography in the 25 asymptomatic patients was a general medical check-up or family screening. Three patients (7.5\%) had a history of unexplained syncope. Although 17 patients (42.5\%) had a history of treated hypertension, blood pressure had been well controlled for $>2$ years in all. One patient $(2.5 \%)$ had a family history of HCM and 3 patients (7.5\%) had a family history of sudden death. All but one patient presented $\mathrm{T}$ wave inversion in anterolateral leads, with a mean maximum negative $T$ wave amplitude of $7.8 \pm 4.2 \mathrm{~mm}$. Of the 39 patients with $\mathrm{T}$ wave inversion on ECG, 12 (30\%) presented giant negative $\mathrm{T}$ waves.

\section{Echocardiography}

Echocardiographic variables are presented in Table 2. In 11 patients referred to our hospital, 2D echocardiogram performed at a local clinic reported mildly increased apical wall thickness $(\geq 12 \mathrm{~mm})$, but it did not reach $15 \mathrm{~mm}$ in most thickened apical segment even in the presence of negative $\mathrm{T}$ wave inversion. Based on echocardiography performed in our hospital, there was no patient who had an LV outflow tract obstruction, either at rest or after provocative valsalva maneuvers. Left atrial dimension was slightly increased $(44.2 \pm 4.8 \mathrm{~mm})$, suggestive of chronic LV diastolic dysfunction. Mean maximal apical wall thickness was $16.7 \pm 2.4 \mathrm{~mm}$. There was no significant correlation between maximal apical wall thickness and the amplitude of $\mathrm{T}$ wave inversion $(\mathrm{r}=0.15, \mathrm{p}=0.34)$ or the sum of $\mathrm{T}$ wave amplitudes in precordial leads $(\mathrm{r}=0.15, \mathrm{p}=0.36)$.

\section{CMR}

In total, 680 segments were analyzed for LV wall thickness and LGE on CMR. Mean LV mass index was $102.2 \pm 28.9 \mathrm{gm} / \mathrm{m}^{2}$ and $\mathrm{LV}$ ejection fraction was $67.7 \pm 8.0 \%$ (Table 3). Maximal apical wall thickness ranged from $15 \mathrm{~mm}$ to $26 \mathrm{~mm}(17.9 \pm 2.3 \mathrm{~mm})$, which was significantly greater than that obtained by echocardiography $(\mathrm{p}<0.01)$. Contrast images demonstrated LGE representing myocardial fibrosis in $30(75.0 \%)$ of the 40 patients and in $130(19 \%)$ of the 680 segments. Total volume of LGE was estimated to be $9.7 \pm 10 \mathrm{~mL}$, corresponding to $4.9 \pm 5.5 \%$ (rage $0.5 \%$ to $26 \%$ ) of total $\mathrm{LV}$ myocardium. The most frequently involved myocardial segments were the apical cap (28 patients $(70 \%))$, followed by the apicolateral segment (20 patients $(52.5 \%)$ ). Of note, LGE was observed not only in the apex of the heart but also in the other segments of the LV, especially in mid anterior and anterolateral segments of the LV and at anterior and posterior attachment points of the right ventricle to the interventricular septum. In addition, LGE was detected in hypertrophied as well as non-hypertrophied areas of the heart. LGE distribution 

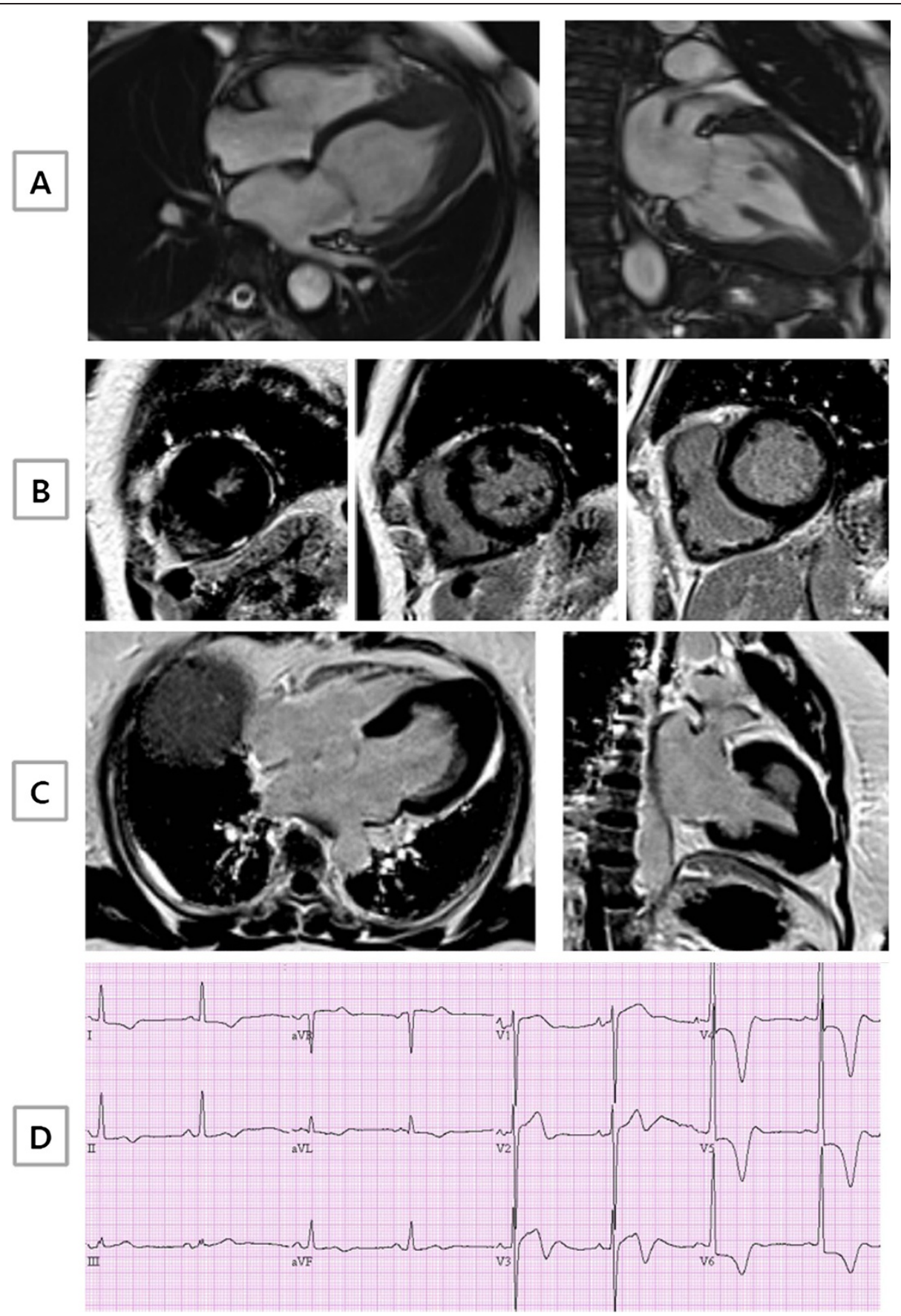

Figure 1 A representative example of ApHCM without late gadolinium enhancement (LGE) by CMR. (A) Long axis CMR cine images clearly demonstrated the presence of apical hypertrophy. However, short (B) and long axis (C) CMR images showed no LGE. (D) Giant negative T wave inversion in anterior leads of electrocardiogram was evident.

is shown schematically in Figure 3. Areas of LGE were transmural ( $\geq 75 \%$ of any segmental wall thickness) in only $2(7 \%)$ of the 30 patients, exclusively limited to the apex and were nontrasmural in the other 28 (93\%) patients, in focal, multifocal or confluent patterns. Interestingly, LGE observed at apical segments was in a "subendocardial" pattern (47 (48\%) of 97 apical segments showing LGE), similar to LGE found in ischemic cardiomyopathy. Representative CMR images of LGE patterns are illustrated in Figure 4.

Although no significant correlation between the depth of $\mathrm{T}$ wave inversion on ECG and maximal apical 


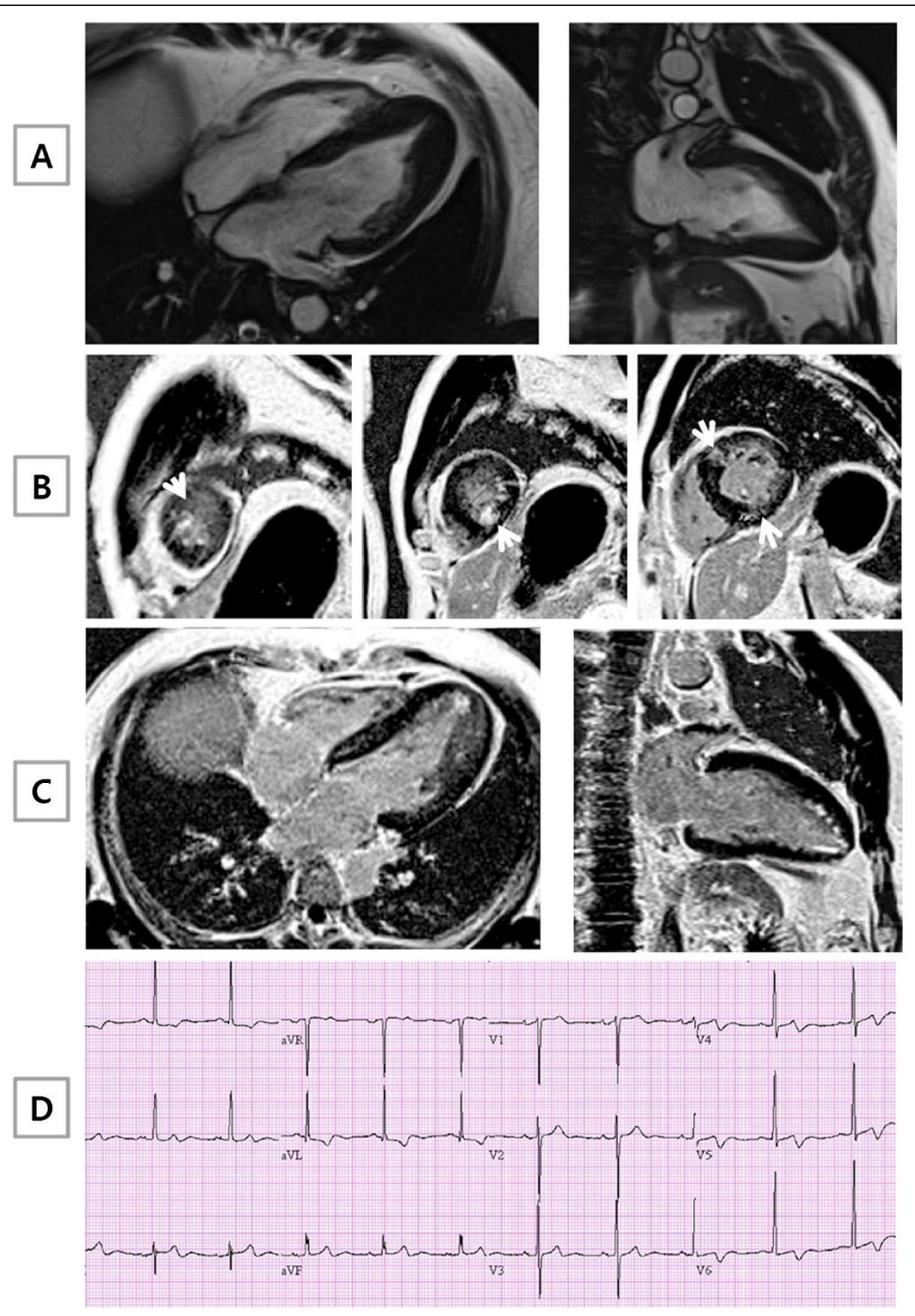

Figure 2 A representative example of ApHCM with late gadolinium enhancement (LGE) by CMR. (A) Long axis CMR cine images clearly demonstrated the presence of apical hypertrophy, as in Figure 1. In contrast, short (B) and long axis (C) CMR images showed diffuse LGE at the basal and mid interventricular septum or RV insertion site (non-hypertrophic segment) and at the apex (hypertrophic segment), as indicated by the arrow. (D) Negative T wave inversion in anterior leads of electrocardiogram was present.

thickness on echocardiography could be demonstrated, maximal apical wall thickness determined by CMR displayed a modest but significant correlation with the amplitude of $\mathrm{T}$ wave inversion on ECG $(\mathrm{r}=0.38$, $\mathrm{p}=0.016)$. An apical aneurysm was detected in 5 patients (12.5\%), and 3 of these (60\%) were not detected by routine echocardiography.

\section{Follow-up}

Over the 2-year follow-up, there was no one who achieved the study end-point, defined a priori, as allcause death, sudden cardiac death and hospitalization for heart failure. Arrhythmic events that were assessed in 23 patients who underwent 24-hour Holter monitoring both at baseline and follow-up were also unremarkable and 
Table 1 Baseline clinical and electrocardiographic characteristics of the study population

\begin{tabular}{lc}
\hline Men/Women & $\mathbf{N}=\mathbf{4 0}$ \\
Age & $31 / 9$ \\
Body surface area $\left(\mathrm{g} / \mathrm{m}^{2}\right)$ & $60.2 \pm 10.4$ \\
Family history of HCM & $1.74 \pm 0.17$ \\
Family history of SCD & $1(2.5 \%)$ \\
History of syncope & $3(7.5 \%)$ \\
NYHA functional class I/II & $3(7.5 \%)$ \\
Hypertension & $25(63.5 \%) / 15(37.5 \%)$ \\
Diabetes mellitus & $17(42.5 \%)$ \\
Smoker & $4(10 \%)$ \\
\end{tabular}

Medications

Calcium-channel blockers
Beta blockers
ACEi/ARB
Aspirin
Diuretics
BP/DBP

$13(32.5 \%)$

$9(22.5 \%)$

$10(25 \%)$

$10(25 \%)$

$6(15 \%)$

$125.9 \pm 13.7 / 75.9 \pm 8.7$

Electrocardiogram

T wave inversion

$39(97.5 \%)$

Giant T waves $\geq 10 \mathrm{~mm}$

$12(30 \%)$

HCM denotes hypertrophic cardiomyopathy; SCD, sudden cardiac death; NYHA, New York Heart Association; ACEi/ARB, angiotension converting esterase inhibitor/angiotensin receptor blocker; S(D)BP, systolic (diastolic) blood pressure; ECG, electrocardiogram.

thus could not be predicted by CMR LGE patterns or its extent, either.

\section{Discussion}

Japanese investigators first reported ApHCM as a morphologic variant of $\mathrm{HCM}$ characterized by a striking

\section{Table 2 Echocardiographic variables}

\begin{tabular}{lc}
\hline LV end-diastolic dimension (mm) & $48.3 \pm 7.2$ \\
LV end-systolic dimension (mm) & $27.3 \pm 4.8$ \\
LV ejection fraction (\%) & $67.7 \pm 7.2$ \\
IVS thickness (mm) & $10.3 \pm 1.6$ \\
PW thickness (mm) & $9.8 \pm 1.3$ \\
Maximal apical wall thickness at end-diastole (mm) & $16.7 \pm 2.4$ \\
LA end-systolic dimension (mm) & $44.2 \pm 4.8$ \\
Peak E wave velocity (m/s) & $0.58 \pm 0.12$ \\
Peak A wave velocity (m/s) & $0.54 \pm 0.16$ \\
Early mitral annular velocity (E', m/s) & $0.11 \pm 0.42$ \\
Mitral inflow/annular velocity ratio (E/E') & $12.96 \pm 4.4$ \\
\hline
\end{tabular}

LV denotes left ventricle; IVS, interventricular septum; PW, posterior wall; and LA, left atrium.
Table 3 CMR findings

\begin{tabular}{lc}
\hline LV end-diastolic volume $(\mathrm{mL})$ & $130.5 \pm 26.3$ \\
LV end-diastolic volume index $\left(\mathrm{mL} / \mathrm{m}^{2}\right)$ & $76.0 \pm 13.9$ \\
LV end-systolic volume $(\mathrm{mL})$ & $43.9 \pm 18.1$ \\
LV end-systolic volume index $\left(\mathrm{mL} / \mathrm{m}^{2}\right)$ & $25.2 \pm 9.1$ \\
LV ejection fraction $(\%)$ & $67.7 \pm 8.0$ \\
LV mass $(\mathrm{gm})$ & $178.0 \pm 61.1$ \\
LV mass index $\left(\mathrm{gm} / \mathrm{m}^{2}\right)$ & $102.2 \pm 28.9$ \\
Maximal apical wall thickness at end-diastole $(\mathrm{mm})$ & $17.9 \pm 2.3$ \\
\hline
\end{tabular}

LV denotes left ventricle.

electrocardiographic pattern of deep $\mathrm{T}$ wave inversion in precordial leads and a distinctive angiographic "ace-ofspades" like appearance of the LV at end-diastole [1416]. Subsequently, echocardiographically detected ApHCM has been repeatedly reported at several centers outside of Japan, although the characteristic electrocardiographic patterns are reported inconsistently in Western patients $[1,17]$. In the present study, in accordance with previous observations $[1,18]$, all but 1 patient (97.5\%) presented with electrocardiographic alterations of ventricular repolarization, in the form of $\mathrm{T}$ wave inversion. The prevalence of a giant negative $T$ wave in our population is also similar to that reported in a Canadian study [1].

Two-dimensional echocardiography is now generally regarded as the "clinical standard" noninvasive diagnostic test for HCM $[19,20]$. However, conventional echocardiography has its technical limitations. As shown in a previous study, it tends to underestimate the magnitude of hypertrophy in the anterolateral free wall, which is frequently spatially away from the center of the echocardiographic sector in parasternal short axis view, resulting in poor lateral resolution, and inconclusive delineation of the epicardial border [21]. Another pitfall of conventional echocardiography is that procurement of apical wall images is not easy due to the close proximity of the echocardiographic probe and the apex, which may result in missing the diagnosis of ApHCM [22,23]. In a previous study, CMR was compared with echocardiography in terms of its ability to detecting hypertrophied segments, and it was concluded that CMR is superior to echocardiography, especially with respect to the detection of apical segmental hypertrophy [24]. In line with findings of the previous study [24], we found that echocardiography significantly underestimated apical wall thickness, as compared with CMR $(16.7 \pm 2.4 \mathrm{~mm}$ vs. $17.9 \pm 2.3 \mathrm{~mm}, \mathrm{p}<0.01)$. Furthermore, echocardiograms performed at a local clinic did not demonstrate sufficient LV apical wall thickness for objective evidence of diagnosing ApHCM in 11 out of 40 patients, that is, in these patients apical wall thickness were $<15 \mathrm{~mm}$ even in the presence of electrocardiographic $\mathrm{T}$ wave inversion, 


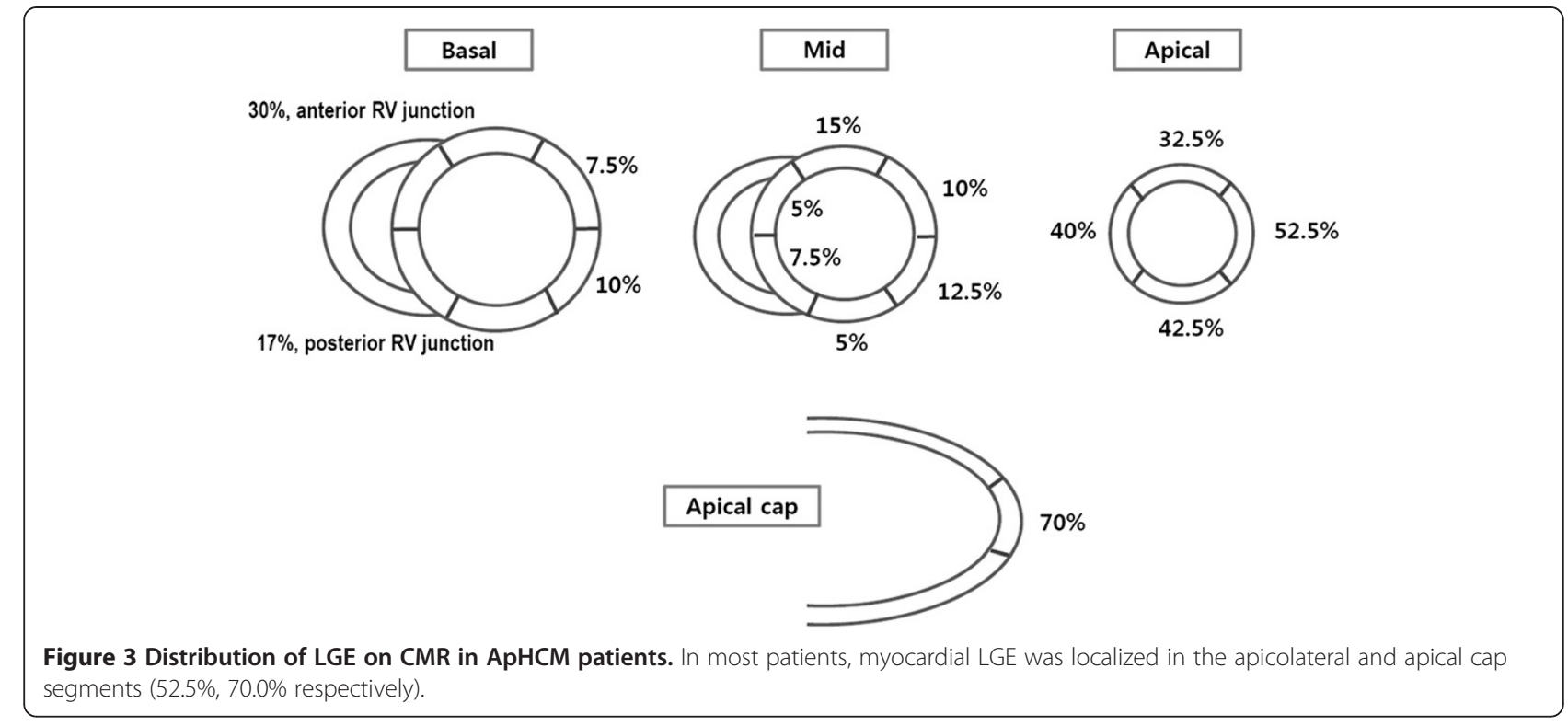

highlighting the advantage of CMR over conventional echocardiography in the accurate delineation of the epicardial border. The advantage of CMR with respect to the visualization of hypertrophied apical LV segments, sometimes not readily identifiable by conventional echocardiography, has been previously demonstrated in
ApHCM [2]. The significant correlation found in the present study between the amplitude of $\mathrm{T}$ wave inversion on ECG and maximal apical wall thickness measured by CMR, but not by echocardiography, confirms the advantage of CMR over echocardiography for the assessments of apical pathology.

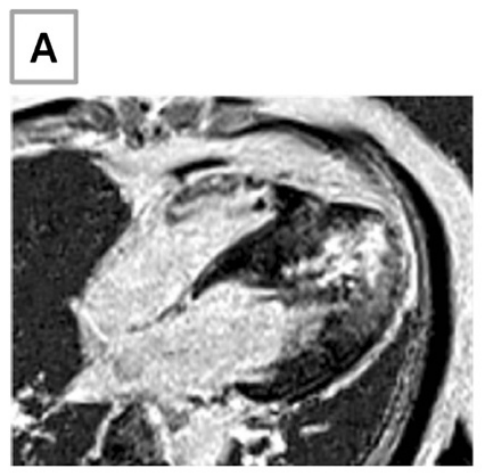

\section{B}
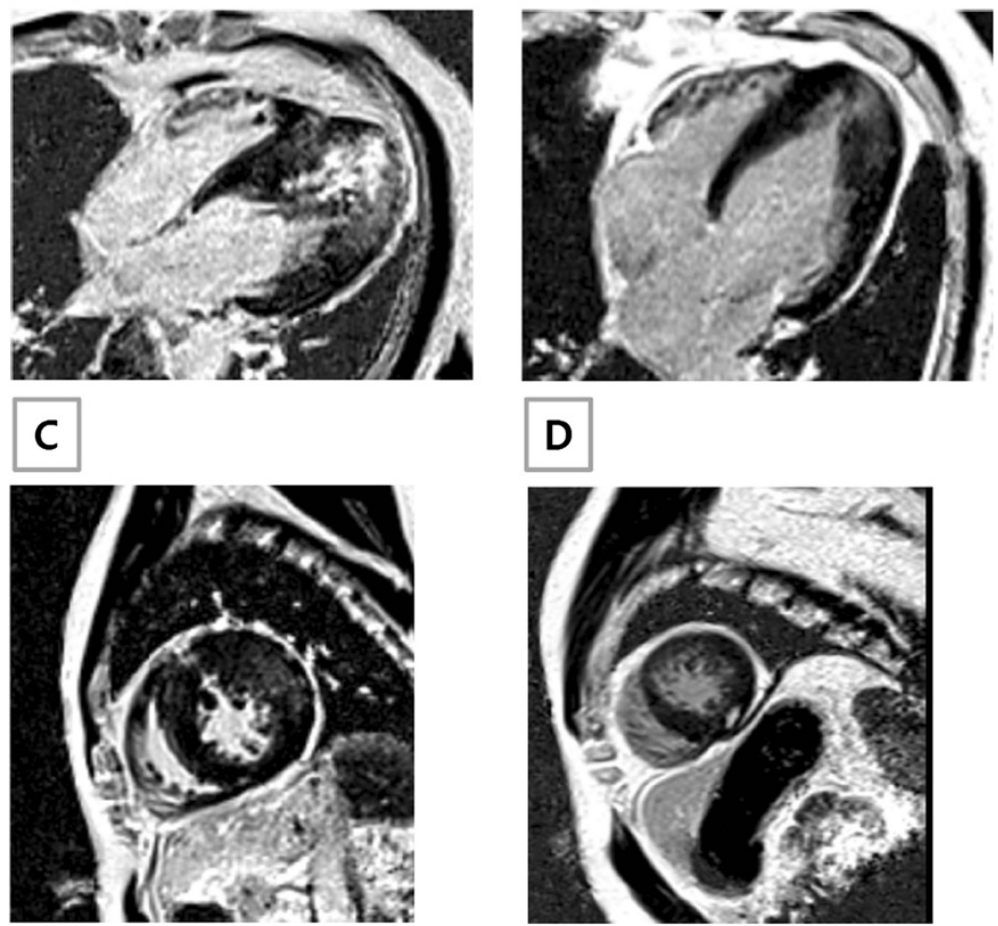

Figure 4 LGE patterns observed in ApHCM patients. (A) Subendocardial LGE with transmurality of $\geq 75 \%$; (B) Fuzzy and confluent LGE; (C) Multifocal LGE at the RV insertion into the septum and mid anterior wall; (D) Discrete (focal) pattern at mid myocardium. 
In the present study, we found that myocardial fibrosis is not an uncommon finding in even asymptomatic or mildly symptomatic 'pure' ApHCM patients. Furthermore, we observed that apical segments, including apical cap, were the most frequently involved LGE sites in ApHCM patients. However, we found that LGE was not limited to hypertrophic apical segments, and that it was also present in basal segments, at the anterior and posterior junctions between the septum and RV free wall, and additionally in non-hypertrophied segments of ApHCM (Figure 3). More importantly, LGE observed at apex (including apical cap) (47 (48\%) of 97 apical segments showing LGE) was in a "subendocardial" pattern, quite unlike the patchy or focal mid-wall LGE pattern frequently observed in asymmetrical septal HCM patients $[4,5,8]$. Given that "ischemic-type" LGE is usually characterized by subendocardial involvement with or without extension into subepicardium [25], the subendocardial LGE pattern at apex in our population strongly suggests previous subendocardial ischemia. Since we exclusively recruited ApHCM patients without significant epicardial coronary artery disease, this "ischemic-type" LGE should be attributable to mechanisms other than epicardial coronary stenosis. A hypertrophied apical wall with relatively deficient coronary blood flow may be a plausible explanation for this "ischemic-type" LGE. However, an increment in intra-apical cavitary pressure that might subsequently lead to suboptimal perfusion to the subendocardial area could explain "ischemic-type" LGE at the apex, as well [26].

Although few studies have addressed LGE in ApHCM, spatial distributions of LGE in ApHCM disagree. In one earlier report, LGE was described only at the apex without any involvement of the base or mid ventricular levels [7], whereas in another report LGE was observed widely in ApHCM patients. More recently, Amano et al. showed the presence of LGE at apex as well as mid-ventricular segments in ApHCM patients [27]. However, they only enrolled symptomatic patients, and thus we still have no data regarding whether LGE is widely distributed even in asymptomatic or minimally symptomatic ApHCM patients. In fact, our study is the largest to investigate the extent and distribution of LGE in an asymptomatic or minimally symptomatic subset of ApHCM, and based on our finding, LGE appears to be distributed across all myocardial segments even in the asymptomatic or minimally symptomatic ApHCM population.

Although ApHCM patients enrolled in the current study were carefully followed for at least 2 years, no one achieved the study end-point (all-cause death, sudden cardiac death or hospitalization for heart failure). No significant arrhythmic events were found, either. Due to the relatively short follow-up period, however, definite conclusions cannot be drawn.
Some limitations of this study warrant consideration. First, the presence of LGE on contrast-enhanced CMR has been well validated in animal models and in humans with ischemic heart disease [28,29]. Although LGE on CMR in HCM patients has been shown to represent increased myocardial collagen in one HCM patient with heart failure, extensive evaluation regarding validation of LGE on CMR has not been systematically performed in ApHCM patients, and thus, the view that LGE exclusively reflects myocardial scarring is, more or less, speculative. Second, the lack of long-term longitudinal followup data prevented definite conclusion of prognostic impact of LGE on CMR in this subset of ApHCM patients. Same caution should be exercised with regard to the prediction of arrhythmic events in this population.

\section{Conclusions}

LGE on CMR is not an uncommon finding in ApHCM patients, and is widely distributed across almost all LV myocardial segments, including non-hypertrophied segments. Given no morbidity or mortality during 2 years of follow-up, it would appear that the simple presence of LGE on CMR is unlikely to be representative of adverse prognosis in this asymptomatic or minimally symptomatic ApHCM population, esp. in the short term.

\section{Abbreviations \\ ApHCM: Apical hypertrophic cardiomyopathy; HCM: Hypertrophic cardiomyopathy; CMR: Cardiovascular magnetic resonance; LGE: Late gadolinium enhancement; LV: Left ventricle.}

\section{Competing interest}

The authors declare that they have no competing interests.

\section{Authors' contributions}

$\mathrm{K}-\mathrm{HK}, \mathrm{H}-\mathrm{KK}, \mathrm{E}-\mathrm{AP}, \mathrm{WL}$, and J-HP contributed to the study design, and performed the analysis. H-KK, S-PL, Y-JK, and D-WS recruited patients and interpreted data. I-CH significantly contributed to LGE assessment of CMR and also interpretation of the data during revision period. $\mathrm{K}-\mathrm{HK}$ and $\mathrm{H}-\mathrm{KK}$ wrote the manuscript. All authors have read and approved the final manuscript.

\section{Funding source}

This study was partly supported by Leading Foreign Research Institute Recruitment Program through the National Research Foundation of Korea (NRF) funded by the Ministry of Education, Science and Technology (MEST) (0640-20100001).

\section{Author details}

${ }^{1}$ Division of Cardiology, Department of Internal Medicine, Cardiovascular Center, Seoul National University College of Medicine, Seoul National University Hospital, 28 Yongon-dong, Chongno-gu, Seoul 110-744, South Korea. ${ }^{2}$ Department of Radiology, Seoul National University College of Medicine, Seoul National University Hospital, 28 Yongon-dong, Chongno-gu, Seoul 110-744, South Korea.

Received: 17 February 2012 Accepted: 10 July 2012

Published: 28 July 2012

\section{References}

1. Eriksson MJ, Sonnenberg B, Woo A, Rakowski P, Parker TG, Wigle ED, Rakowski H: Long-term outcome in patients with apical hypertrophic cardiomyopathy. J Am Coll Cardiol. 2002, 39:638-645. 
2. Moon JC, Fisher NG, McKenna WJ, Pennell DJ: Detection of apical hypertrophic cardiomyopathy by cardiovascular magnetic resonance in patients with non-diagnostic echocardiography. Heart. 2004, 90:645-649.

3. Moon JC, Reed E, Sheppard MN, Elkington AG, Ho SY, Burke M, Petrou M, Pennell DJ: The histologic basis of late gadolinium enhancement cardiovascular magnetic resonance in hypertrophic cardiomyopathy. J Am Coll Cardiol. 2004, 43:2260-2264.

4. Choudhury L, Mahrholdt H, Wagner A, Choi KM, Elliott MD, Klocke FJ, Bonow RO, Judd RM, Kim RJ: Myocardial scarring in asymptomatic or mildly symptomatic patients with hypertrophic cardiomyopathy. J Am Coll Cardiol. 2002, 40:2156-164.

5. Amano Y, Takayama M, Takahama K, Kumazaki T: Delayed hyperenhancement of myocardium in hypertrophic cardiomyopathy with asymmetrical septal hypertrophy: comparison with global and regional cardiac MR imaging appearances. J Magn Reson Imaging. 2004, 20:595-600

6. Kwon DH, Smedira NG, Rodriguez ER, Tan C, Setser R, Thamilarasan M, Lytle BW, Lever HM, Desai MY: Cardiac magnetic resonance detection of myocardial scarring in hypertrophic cardiomyopathy: correlation with histopathology and prevalence of ventricular tachycardia. J Am Coll Cardiol. 2009, 54:242-249.

7. Yamada M, Teraoka K, Kawade M, Hirano M, Yamashina A: Frequency and distribution of late gadolinium enhancement in magnetic resonance imaging of patients with apical hypertrophic cardiomyopathy and patients with asymmetrical hypertrophic cardiomyopathy: a comparative study. Int J Cardiovasc Imaging. 2009, 25(Suppl 1):131-138.

8. Fattori R, Biagini E, Lorenzini M, Buttazzi K, Lovato L, Rapezzi C: Significance of magnetic resonance imaging in apical hypertrophic cardiomyopathy. Am J Cardiol. 2010, 105:1592-1596.

9. Choi EY, Rim SJ, Ha JW, Kim YJ, Lee SC, Kang DH, Park SW, Song JK, Sohn DW, Chung N: Phenotypic spectrum and clinical characteristics of apical hypertrophic cardiomyopathy: multicenter echo-Doppler study. Cardiology. 2008, 110:53-61.

10. Chang SA, Kim HK, Kim DH, Kim JC, Kim YJ, Kim HC, Sohn DW, Oh BH, Park YB: Left ventricular twist mechanics in patients with apical hypertrophic cardiomyopathy: assessment with 2D speckle tracking echocardiography. Heart. 2010, 96:49-55.

11. Cerqueira MD, Weissman NJ, Dilsizian V, Jacobs AK, Kaul S, Laskey WK, Pennell DJ, Rumberger JA, Ryan T, Verani MS: American Heart Association Writing Group on Myocardial Segmentation and Registration for Cardiac Imaging. Standardized myocardial segmentation and nomenclature for tomographic imaging of the heart: a statement for healthcare professionals from the Cardiac Imaging Committee of the Council on Clinical Cardiology of the American Heart Association. Circulation. 2002, 105:539-542.

12. Adabag AS, Maron BJ, Appelbaum E, Harrigan CJ, Buros JL, Gibson CM, Lesser JR, Hanna CA, Udelson JE, Manning WJ: Occurrence and frequency of arrhythmias in hypertrophic cardiomyopathy in relation to delayed enhancement on cardiovascular magnetic resonance. J Am Coll Cardiol. 2008, 51:1369-1374.

13. Nazarian S, Bluemke DA, Lardo AC, Zviman MM, Watkins SP, Dickfeld TL, Meininger GR, Roguin A, Calkins H, Tomaselli GF, Weiss RG, Berger RD, Lima JA, Halperin HR: Magnetic resonance assessment of the substrate for inducible ventricular tachycardia in nonischemic cardiomyopathy. Circulation. 2005, 112:2821-2825.

14. Sakamoto T, Tei C, Murayama M, Ichiyasu H, Hada Y: Giant t wave inversion as a manifestation of asymmetrical apical hypertrophy (aah) of the left ventricle. Echocardiographic and ultrasono-cardiotomographic study. Japanese Heart Journal. 1976, 17:611.

15. Yamaguchi H, Ishimura T, Nishiyama S, Nagasaki F, Nakanishi S, Takatsu F, Nishijo T, Umeda T, Machii K: Hypertrophic nonobstructive cardiomyopathy with giant negative t waves (apical hypertrophy): Ventriculographic and echocardiographic features in 30 patients. Am J Cardiol. 1979, 44:401-412

16. Kitaoka H, Doi Y, Casey SA, Hitomi N, Furuno T, Maron BJ: Comparison of prevalence of apical hypertrophic cardiomyopathy in Japan and the United States. Am J Cardiol. 2003, 92:1183-1186.

17. Maron BJ, Bonow RO, Seshagiri TN, Roberts WC, Epstein SE: Hypertrophic cardiomyopathy with ventricular septal hypertrophy localized to the apical region of the left ventricle (apical hypertrophic cardiomyopathy). Am J Cardiol. 1982, 49:1838-1848.
18. Suzuki J, Watanabe F, Takenaka K, Amano K, Amano W, Igarashi T, Aoki T, Serizawa T, Sakamoto T, Sugimoto T, et al: New subtype of apical hypertrophic cardiomyopathy identified with nuclear magnetic resonance imaging as an underlying cause of markedly inverted T waves. J Am Coll Cardiol. 1993, 22:1175-1181.

19. Maron BJ: Hypertrophic cardiomyopathy: a systematic review. JAMA. 2002, 287:1308-1320.

20. Maron BJ, McKenna WJ, Danielson GK, Kappenberger LJ, Kuhn HJ, Seidman CE, Shah PM, Spencer WH 3rd, Spirito P, Ten Cate FJ, Wigle ED: Task Force on Clinical Expert Consensus Documents. American College of Cardiology; Committee for Practice Guidelines. European Society of Cardiology. American College of Cardiology/European Society of Cardiology clinical expert consensus document on hypertrophic cardiomyopathy. A report of the American College of Cardiology Foundation Task Force on Clinical Expert Consensus Documents and the European Society of Cardiology Committee for Practice Guidelines. J Am Coll Cardiol. 2003, 42:1687-1713.

21. Rickers C, Wilke NM, Jerosch-Herold M, Casey SA, Panse P, Panse N, Weil J, Zenovich AG, Maron BJ: Utility of cardiac magnetic resonance imaging in the diagnosis of hypertrophic cardiomyopathy. Circulation. 2005, 112:855-861.

22. Suzuki Y, Kadota K, Nohara R, Tamaki S, Kambara H, Yoshida A, Murakami T, Osakada G, Kawai C, Tamaki N, et al: Recognition of regional hypertrophy in hypertrophic cardiomyopathy using thallium-201 emission-computed tomography: comparison with two-dimensional echocardiography. Am J Cardiol. 1984, 53:1095-1102.

23. Casolo GC, Trotta F, Rostagno C, Poggesi L, Galanti G, Masotti G, Bartolozzi C, Dabizzi RP: Detection of apical hypertrophic cardiomyopathy by magnetic resonance imaging. Am Heart J. 1989, 117:468-472.

24. Pons-Lladó G, Carreras F, Borrás X, Palmer J, Llauger J, Bayés de Luna A: Comparison of morphologic assessment of hypertrophic cardiomyopathy by magnetic resonance versus echocardiographic imaging. Am J Cardiol 1997, 79:1651-1656.

25. Mahrholdt H, Wagner A, Judd RM, Sechtem U, Kim RJ: Delayed enhancement cardiovascular magnetic resonance assessment of nonischaemic cardiomyopathies. Eur Heart J. 2005, 26:1461-1474.

26. Matsubara K, Nakamura T, Kuribayashi T, Azuma A, Nakagawa M: Sustained cavity obliteration and apical aneurysm formation in apical hypertrophic cardiomyopathy. J Am Coll Cardiol. 2003, 42:288-295.

27. Amano Y, Takayama M, Fukushima Y, Kitamura M, Kumita S: Delayedenhancement MRI of apical hypertrophic cardiomyopathy: assessment of the intramural distribution and comparison with clinical symptoms, ventricular arrhythmias, and cine MRI. Acta Radiol. 2001, 52:613-8.

28. Wu E, Judd RM, Vargas JD, Klocke FJ, Bonow RO, Kim RJ: Visualisation of presence, location, and transmural extent of healed Q-wave and non-Q-wave myocardial infarction. Lancet. 2001, 357:21-28.

29. Kim RJ, Fieno DS, Parrish TB, Harris K, Chen EL, Simonetti O, Bundy J, Finn JP, Klocke FJ, Judd RM: Relationship of MRI delayed contrast enhancement to irreversible injury, infarct age, and contractile function. Circulation 1999, 100:1992-2002.

doi:10.1186/1532-429X-14-52

Cite this article as: Kim et al:: Myocardial scarring on cardiovascular magnetic resonance in asymptomatic or minimally symptomatic patients with "pure" apical hypertrophic cardiomyopathy. Journal of Cardiovascular Magnetic Resonance 2012 14:52. 